\title{
Analysis of the use of blood parameters in COPD and ACOS for the purposes of disease differentiation
}

\author{
DUYGU ZORLU
}

\begin{abstract}
Department of Pulmonology, Faculty of Medicine, Ahi Evran University, Kirsehir 40100, Turkey
\end{abstract}
Received March 30, 2021; Accepted October 12, 2021

DOI: $10.3892 /$ wasj.2021.133

\begin{abstract}
The definition of asthma-chronic obstructive pulmonary disease (COPD) overlap syndrome (ACOS) has recently been changed to 'ACO' in cases in which certain clinical manifestations of both asthma and COPD are present. In ACO, difficulties in diagnosis and differential diagnosis occur, since inflammation and related pathophysiological changes cannot be clearly demonstrated. In the present study, blood parameters were evaluated with regards to the differential diagnosis of ACO-COPD and are presented with the aim of providing an approach that is easy to apply in daily practice. The present study was conducted in February, 2020 on patients who presented to the pulmonology department. A total of 50 patients with COPD and 51 patients with ACO who were newly diagnosed were included in the study. The results revealed that there were significant differences between the ACO and COPD groups in terms of their neutrophil and lymphocyte counts, platelet (PLT) and C-reactive protein (CRP) counts, neutrophil to lymphocyte ratio (NLR) and platelet to lymphocyte ratio $(\mathrm{PLR})(\mathrm{P}<0.05)$. However, no statistically significant differences were found between the groups in terms of their eosinophil ratio and mean platelet volume (MPV) count ( $\mathrm{P}>0.05)$. The mean neutrophil count, NLR, PLR and CRP values were significantly higher in the COPD group than the ACO group. In the ACO group, the mean PLT and lymphocyte count values were significantly higher than those in the COPD group. According to the findings of the present study, although role of inflammation in ACO has not been fully clarified, the presence of eosinophilia in ACO does not significantly contribute to the differential diagnosis of COPD. MPV also lacks clinically significant differential properties in COPD and ACO. However, the mean PLT and lymphocyte counts were significantly higher in the ACO than in the COPD group, which maybe a promising result in the
\end{abstract}

Correspondence to: Dr Duygu Zorlu, Department of Pulmonology, Faculty of Medicine, Ahi Evran University, Kervansaray, 2019. Sk. No. 1, Kirsehir 40100, Turkey

E-mail: trbzorlu@yahoo.com; duygu.karayigit@ahievran.edu.tr

Key words: asthma-chronic obstructive pulmonary disease overlap syndrome, blood biomarkers, inflammation differential diagnosis of COPD. Thus, the present study may provide insight into the differential diagnosis between ACO and COPD.

\section{Introduction}

The diagnosis of chronic obstructive pulmonary disease (COPD) should be considered in patients with dyspnea, chronic cough and/or sputum expectorating complaints and/or in individuals exposed to risk factors for the disease, and spirometry is considered necessary for diagnosis (1). A post-bronchodilator first second Force Expiratory Volume (FEV1)/forced vital capacity (FVC) $<70 \%$ indicates persistent airflow limitation and allows for a diagnosis to be made in the presence of appropriate symptoms and exposure. Chronic and progressive dyspnea, cough and sputum are the most characteristic symptoms of COPD. These symptoms vary daily and may precede the development of airflow limitation over the years $(2,3)$.

Asthma-COPD overlap syndrome (ACOS) was objectively defined for the first time in the Global Initiative for Asthma (GINA) and Global Initiative for Obstructive Lung Disease (GOLD) 2014 guidelines as 'Asthma and COPD features accompanying each other at similar rates in cases with persistent airflow limitation' (4). The diagnosis of ACOS should be considered in patients with persistent airflow limitation (post-bronchodilator FEV1/FVC $<70 \%$ ), in asthmatic patients with a history of smoking or in patients with COPD with a history of childhood asthma experiencing eosinophilia and a reversibility in pulmonary function tests (PFTs) (5). The symptoms of these patients indicate a chronic airway disease; however, the sensitivity and specificity of their symptoms and characteristics are low in the differential diagnosis of asthma, ACOS or COPD. In the GINA-GOLD joint guidelines, it is also stated that those who have three or more of the characteristics of asthma and COPD for each can be defined as suffering from ACOS (4-6).

In epidemiological studies, the prevalence of ACOS has been reported as $15-20 \%$. This rate varies according to the asthma and COPD diagnostic criteria in the studies and the age groups of the patients examined. In a previous review study, the prevalence of ACOS was shown to be between 12.1 and $55.2 \%$ among patients with COPD and between 13.3 and $61.0 \%$ among patients with asthma alone (7). In another, multicenter, cross-sectional, observational study, the number of patients 
with ACOS was found to be $59 \%$ (8). In questionnaires conducted on 3,500 participants in the Wellington Respiratory Study, $20 \%$ of the patients who were evaluated using tomography, PFTs or both criteria were diagnosed with COPD, and since $55 \%$ exhibited additional clinical features of asthma, they were defined as having ACOS, which presents a common clinical image (9). Therefore, standardization has not yet been achieved in the diagnosis of ACO. The definition of ACOS has recently been changed to 'ACO' in cases where some clinical manifestations of both Asthma and COPD are present. It is stated that those who have three or more of the features of asthma and COPD for each can be defined as ACO (4-6). Thus, the term ACO is used henceforth.

The aim of the present study was to enhance the understanding, management and follow-up of ACO, by evaluating blood parameters [neutrophil to lymphocyte ratio (NLR), mean platelet volume (MPV), eosinophil count and platelet to lymphocyte ratio (PLR)] that can be easily used in daily practice for the diagnosis of ACO, and as a simple method which can be used to differentiate COPD from ACO.

\section{Patients and methods}

Study approval. Ethics committee approval for the study was obtained from the Ahi Evran University Faculty of Medicine Clinical Research Ethics Committee with (approval no. 2019-04/46). Verbal and written consent was obtained from each patient who volunteered to participate in the study.

Study population. The present study was planned prospectively and was conducted on patients who were newly diagnosed with COPD and ACO and admitted to the Pulmonology Outpatient Clinic, Ahi Evran University Research and Training Hospital, Kirsehir, Turkey in February, 2020. There were 676 patients in total who presented to the Pulmonology Outpatient Clinic in February, 2020. Of these patients, 148 were newly diagnosed with COPD, and 134 were newly diagnosed with ACO. The patients were diagnosed with COPD and ACO according to the criteria specified in the GINA and GOLD guidelines. In total, 35 patients with COPD and 20 patients with ACO with additional diseases, such as diabetes mellitus (DM), essential hypertension (HT), and cardiovascular, gastrointestinal, central nervous system and respiratory diseases, as well as 40 patients with COPD and 22 patients with ACO with a history of any drug use were excluded from the study. Patients with a history of smoking (one pack for $\geq 1$ year) and patients with obstruction indicated by the PFTs (post-bronchodilator FEV1/FVC $<70 \%$ ) were included in the study. Patients with a normal posteroanterior (PA) chest radiograph were excluded from the study. In total, 15 patients with COPD and 17 patients with ACO without a history of smoking at the time of admission, and 5 patients with COPD and 19 patients ACO with a normal PA chest X-ray who were newly diagnosed were also excluded from the study. In addition, 2 patients with ACO and 3 patients with COPD did not agree to participate in the study; 3 patients ACO later stated that they did not wish to be included in the study, and these patients were thus also excluded from the study.

Thus, a total of 101 volunteering newly diagnosed patients (50 patients with COPD and 51 patients with ACO), without a history of inhaler and/or any drug use and with no comorbidities, with a history of smoking one pack every day for $\geq 1$ year and/or occupational exposure to environmental pollutants/carcinogens (such as in the cast iron casting, tire and cement factories) with radiological findings on direct X-ray and complete obstruction in PFTs, were included in the study.

A single pulmonologist examined the patients who were included in the study. After recording information on the demographic characteristics and clinical parameters of the patients, blood samples from the patients were immediately transferred to the biochemistry laboratory. Complete blood count tests from blood drawn into K2EDTA tubes were performed using the Sysmex XN-1000 (Sysmex Corporation) automatic blood count device. In these routine blood analyses, MPV values and eosinophil counts were recorded from the hemogram results. NLR and PLR were calculated by the one-to-one proportioning of the neutrophil, lymphocyte and platelet counts in the blood.

Statistical analysis. In the power analysis that was conducted, the effect size was taken as $d=0.60$, with $\alpha=0.05$ and Power( $1-\beta)$ err prob $=0.85$. As a result of the power analysis, it was calculated that the study needed to include a minimum of 101 participants. At the first stage, the Shapiro-Wilk test was applied for all medical parameters on the basis of the ACO and COPD groups, and it was observed that other parameters, apart from the eosinophil counts, NLR, PLR and C-reactive protein (CRP) values, met the normality assumption on the basis of the groups. However, since the number of observations on a group basis was high $(n>30)$, parametric hypothesis tests could be applied even when the normal distribution condition was not met. The Chi-squared test was used for the data for sex and the t-test was used for all other data. Statistical analyses were carried out using the IBM SPSS 20 bundle software (IBM Corp.).

\section{Results}

A total of 101 patients were included in the study. Of these patients, 50 were newly diagnosed with COPD, and 51 were newly diagnosed with ACO. According to the demographic data, $58.4 \%$ of the participants were male, and $41.6 \%$ were female. The mean age of the patients was 63 years. In total, $50.5 \%$ of the patients were in the ACO group, and $49.5 \%$ were in the COPD group. In addition, $27.5 \%$ of the patients in the ACO group were male, $72.5 \%$ were female, and the mean age of this group was 58 years. In the COPD group, $90 \%$ of the patients were male, $10 \%$ were female, and the mean age of this group was 68 years (Table I).

According to the results of the independent-samples t-test of the parameters between the ACO and COPD groups, there was a statistically significant difference between the ACO and COPD groups in terms of neutrophil and lymphocyte values, platelet (PLT) and CRP counts, NLR and PLR $(\mathrm{P}<0.05)$. However, no statistically significant differences were observed between the groups in terms of their eosinophil counts and MPV values ( $\mathrm{P}>0.05)$ (Tables I and II).

The mean neutrophil counts, NLR, PLR and CRP values were significantly higher in the COPD group than the ACO group. In the ACO group, the mean PLT value and lymphocyte count were significantly higher than those in the COPD group. 
Table I. Demographic and blood parameter characteristics of the patients in the ACO and COPD groups.

\begin{tabular}{|c|c|c|c|}
\hline Variable & $\operatorname{ACO}(n=51)$ & $\operatorname{COPD}(\mathrm{n}=50)$ & P-value \\
\hline Age, years & $58.4 \pm 10.2$ & $68.1 \pm 7.5$ & $<0.001^{\mathrm{c}}$ \\
\hline \multicolumn{4}{|l|}{ Sex, n (\%) } \\
\hline Male & $14(27.5)$ & $45(90)$ & \multirow[t]{2}{*}{$<0.001^{\mathrm{c}}$} \\
\hline Female & $37(72.5)$ & $5(10)$ & \\
\hline \multicolumn{4}{|l|}{ Blood parameters } \\
\hline Neutrophil \% & $57.2 \pm 8.9$ & $66.9 \pm 12.8$ & $<0.001^{\mathrm{c}}$ \\
\hline Lymphocyte $\%$ & $31.7 \pm 8.5$ & $21.3 \pm 11.3$ & $<0.001^{\mathrm{c}}$ \\
\hline Eosinophil \% & $3.1 \pm 3.3$ & $2.2 \pm 2.4$ & 0.092 \\
\hline Eosinophil & $0.27 \pm 0.34$ & $0.19 \pm 0.20$ & 0.136 \\
\hline PLT & $272.3 \pm 60.4$ & $238.1 \pm 65.7$ & $0.008^{\mathrm{b}}$ \\
\hline MPV & $10.2 \pm 1$ & $10.1 \pm 0.8$ & 0.899 \\
\hline CRP & $1 \pm 1.5$ & $4.5 \pm 8.7$ & $0.010^{\mathrm{a}}$ \\
\hline Neutrophil/lymphocyte & $2.2 \pm 2$ & $5.3 \pm 5.6$ & $<0.001^{\mathrm{c}}$ \\
\hline PLT/lymphocyte & $110.4 \pm 36.9$ & $146.3 \pm 78.7$ & $0.005^{\mathrm{b}}$ \\
\hline Eosinophil/lymphocyte & $0.1 \pm 0.1$ & $0 \pm 0$ & $0.042^{\mathrm{c}}$ \\
\hline
\end{tabular}

COPD, chronic obstructive pulmonary disease; ACO, asthma-COPD overlap syndrome; PLT, platelet; MPV, mean platelet volume; CRP, $\mathrm{C}$-reactive protein. Statistically significant differences are indicated as follows: ${ }^{a} \mathrm{P}<0.05,{ }^{\mathrm{b}} \mathrm{P}<0.01$ and ${ }^{\mathrm{c}} \mathrm{P}<0.001$.

Table II. Results of comparisons of the of parameters between the ACO and COPD groups using an independent samples t-test.

\begin{tabular}{|c|c|c|c|c|c|c|c|c|}
\hline Variable & Group & No. of patients & Mean & SD & Minimum & Maximum & t-value & P-value \\
\hline \multirow[t]{2}{*}{ Neutrophil (\%) } & $\mathrm{ACO}$ & 51 & 57.208 & 8.907 & 39.800 & 88.500 & $-4.402^{c}$ & $<0.001$ \\
\hline & COPD & 50 & 66.886 & 12.803 & 38.400 & 92.100 & & \\
\hline \multirow[t]{2}{*}{ Lymphocyte (\%) } & $\mathrm{ACO}$ & 51 & 31.678 & 8.505 & 5.900 & 49.900 & $5.221^{\mathrm{c}}$ & $<0.001$ \\
\hline & COPD & 50 & 21.256 & 11.330 & 3.200 & 53.800 & & \\
\hline \multirow[t]{2}{*}{ Eosinophil (\%) } & ACOS ACO & 50 & 3.136 & 3.259 & 0.000 & 20.300 & 1.699 & 0.092 \\
\hline & COPD & 50 & 2.162 & 2.410 & 0.000 & 15.000 & & \\
\hline \multirow[t]{2}{*}{ PLT } & $\mathrm{ACO}$ & 51 & 272.294 & 60.368 & 107.000 & 368.000 & $2.728^{\mathrm{b}}$ & 0.008 \\
\hline & COPD & 50 & 238.060 & 65.674 & 78.000 & 413.000 & & \\
\hline \multirow[t]{2}{*}{ MPV } & $\mathrm{ACO}$ & 50 & 10.158 & 1.000 & 8.300 & 12.700 & 0.127 & 0.899 \\
\hline & COPD & 49 & 10.135 & 0.823 & 8.600 & 12.800 & & \\
\hline \multirow[t]{2}{*}{ CRP } & $\mathrm{ACO}$ & 35 & 0.984 & 1.475 & 0.020 & 7.800 & $-2.668^{a}$ & 0.010 \\
\hline & COPD & 46 & 4.468 & 8.694 & 0.050 & 48.090 & & \\
\hline \multirow[t]{2}{*}{ Neutrophil/lymphocyte } & $\mathrm{ACO}$ & 51 & 2.152 & 1.983 & 0.831 & 15.082 & $-3.764^{c}$ & $<0.001$ \\
\hline & COPD & 50 & 5.296 & 5.571 & 0.713 & 27.720 & & \\
\hline \multirow[t]{2}{*}{ PLT/lymphocyte } & $\mathrm{ACO}$ & 51 & 110.395 & 36.935 & 50.743 & 208.235 & $-2.922^{b}$ & 0.005 \\
\hline & COPD & 50 & 146.265 & 78.738 & 9.949 & 434.667 & & \\
\hline
\end{tabular}

COPD, chronic obstructive pulmonary disease; ACO, asthma-COPD overlap syndrome; PLT, platelet; MPV, mean platelet volume; SD, standard deviation; CRP, C-reactive protein. Statistically significant differences are indicated as follows: ${ }^{a} \mathrm{P}<0.05,{ }^{\mathrm{b}} \mathrm{P}<0.01$ and ${ }^{\mathrm{c}} \mathrm{P}<0.001$.

\section{Discussion}

Over the years, NLR values, PLR values and eosinophil counts have been evaluated for their usability as markers of inflammation in a wide variety of disease groups in daily practice (4,10-15). A number of studies have been conducted for this purpose in COPD, and these markers have begun to be used in daily practice in relation to COPD (14-19). Studies on this topic in ACO are still very limited. Therefore, the present study was conducted in order to determine the effectiveness of these markers (blood parameters) in the diagnosis of ACO, as well as whether they can be used in clinical practice and 
to facilitate follow-up. The present study also wished to determine whether these markers can be used to differentiate COPD from ACO.

The definition of ACOS has recently been changed to 'ACO' in cases where some clinical manifestations of both Asthma and COPD are present. It is stated that those who have three or more of the features of asthma and COPD for each can be defined as ACO (4-6). The main diagnostic criteria of ACO are patients who are not fully responsive to bronchodilation tests, have been exposed to smoking and/or biomass and present with airflow obstruction with an incomplete history of concomitant atopy or asthma. The condition corresponds to the presence of eosinophilic airway and systemic inflammation, a positive response to corticosteroid therapy and a high concentration of exhaled nitric oxide in additional diagnostic evaluation. Due to airway remodeling, ACO differs from asthma with bronchial obstruction that is not completely reversible. However, the role of systemic inflammation in ACO has not yet been fully determined. Inflammation is the common point in the pathogenesis of both COPD and asthma. However, the mechanisms and duration of inflammation are the reasons for the clinicopathological differences. In previous studies, in peripheral blood, as a reflection of inflammation, the NLR and PLR was found to be significantly increased in COPD and asthma, and thus, they are significant parameters in this respect (4-6).

The results of the present study also demonstrated a statistically significant difference between the ACO and COPD groups in terms of their neutrophil and lymphocyte counts, PLT and CRP counts, NLR and PLR $(\mathrm{P}<0.05)$. However, no statistically significant differences were found between the two groups in terms of their eosinophil counts and MPV $(\mathrm{P}>0.05)$. The mean neutrophil count, NLR, PLR and CRP values were significantly higher in the COPD group than the ACO group. In the ACO group, the mean PLT and lymphocyte counts were significantly higher than those in the COPD group. In the present study, no significant differences were found as regards eosinophilia in the $\mathrm{ACO}$ and COPD groups in the present study. In other words, eosinophilia cannot provide the expected use in the differential diagnosis of ACO and COPD.

In the Gene-Environment Interactions in Respiratory Diseases (GEIRD) study $(n=8,360)$, the physician-diagnosed ACO rate was $1.6 \%$ in patients at the ages of 20-44 years, $2.1 \%$ in those at the ages of 45-64 years and $4.5 \%$ in those at the ages of 65-84 years (17). In addition, among the 915 patients with COPD included in the multicenter COPD gene study investigating the genetic basis and phenotypes of COPD, 119 (13\%) cases were identified as having ACOS (20). In a previous study conducted in Turkey, the rate of ACO was reported as $31.5 \%$ (21). Furthermore, in a meta-analysis based on population-based studies, the global prevalence of ACO was estimated as $2.0 \%$; however, it was emphasized that the prevalence of $\mathrm{ACO}$ depends on the diagnostic criteria. Therefore, there is a vital need to better define the diagnostic criteria, management and treatment of $\operatorname{ACO}(22,23)$. Another study also found that the prevalence of ACO varied widely worldwide from 0.9 to $11.1 \%$ in the general population, from 11.1 to $61.0 \%$ in patients with asthma and from 4.2 to $66 \%$ in patients with COPD. Additionally, it was emphasized that the frequency of exacerbation and the prognosis of patients with ACO were not clearly demonstrated (24). In the present study, the incidence of ACO was found to be $19.8 \%$ (134/676 patients).

In a study conducted in a severe asthma clinic, the prevalence of ACOS was reported to be half of the prevalence of severe asthma cases (10). It has been suggested that PFT should be considered if a patient with COPD has reversible airway obstruction $(>12 \%$ and $>200 \mathrm{ml}$ increase in FEV1 compared to the baseline after $400 \mu \mathrm{g}$ salbutamol or $1,000 \mu \mathrm{g}$ terbutaline application), and if persistent airflow limitation is present in a patient with asthma, ACO should be considered $(5,24,25)$. It has been stated that a low diffusing capacity of the lungs for carbon monoxide (DLCO), static hyperinflation findings, bronchial hypersensitivity and peak expiratory flow (PEF) variability may accompany functional status in these cases, but they are not necessarily present $(9,10,12,22,26-28)$. Therefore, evaluation parameters other than PFT were not used in the present study.

When evaluated in terms of radiological features, there appears to be limited data on the radiological features of ACO. In these limited data, it has been shown that radiological findings may not be distinctive in cases with ACO, particularly at the early stages. However, it has been stated that, as the severity of the disease increases, hyperinflation, an increase in airway wall thickness, air trapping, increased transparency, bullae or emphysematous changes may also be observed $(4,5,29)$. Hence, radiological findings are similar to those with severe asthma and COPD. In the present study, patients with any of these radiological findings were evaluated, and patients without radiological findings were excluded from the study.

According to the results of the present study, there was a predominantly lymphocytic response in ACO. Of note, contrary to what is known and stated in the guidelines, no significant difference was found in the eosinophilic response in ACO. This situation may be interpreted as that eosinophilia is not observed intensely in ACO, and/or it does not make a significant difference when observed (4). This interpretation may be evaluated as a warning that it does not provide the expected use in the differential diagnosis of ACO, as it does not contribute to the diagnosis and follow-up. This view is also shared by other researchers, in line with the results of the present study (30). As neutrophils and platelets are the blood cell group mainly responsible for inflammation, the levels of these demonstrated in a significant proportional difference in COPD, as was expected.

As also demonstrated herein, in daily practice, MPV is not a marker supporting the diagnosis and differential diagnosis of ACO or COPD. Perhaps, evaluating MPV according to COPD stages may result in a significant difference depending on the chronic process. For this purpose, further studies are required on this matter. Nevertheless, with the available data, no significance could be determined in the present study.

Notably, in the GOLD 2019 update (4), ACO was not yet mentioned among the disease groups with differential diagnosis of COPD. The reason for this may be that ACO is currently considered as a clinical definition. The lack of a common definition in a number of studies may also be due to this issue $(5,7,8)$. The treatment and follow-up of many cases continue, and a related guideline update is expected in the near future. Therefore, in a previous study, the researchers stated 
that they determined their own objective criteria, and these criteria should be included in the guidelines. In COPD, due to alveolar wall destruction, it was stated that the DLCO and CT results were strongly associated, while similarly, atopic tendency and a high fractional exhaled nitric oxide (FeNO) were stated to be useful features for the diagnosis of airway eosinophilic inflammation asthma (30). In another study, in a spirometric evaluation that can be useful for diagnosis, pre-bronchodilator FEV1/FVC was found to be significantly lower in the ACO group, although no significant difference was found in the post-bronchodilator group (31).

Since the severity of the inflammatory response would increase in individuals with comorbid diseases or in individuals with two or more inflammatory diseases, it is difficult to determine the limit numerical values of the aforementioned proportional values. Limited studies are available comparing these inflammatory proportional values in COPD and ACO. Since additional diseases causing inflammation were excluded from the present study, it is considered that the current inflammation was only related to primary diseases (COPD or ACO), and the present results on this topic are valuable.

In fact, the available data demonstrate that the treatment of patients with COPD and ACO does not exhibit differences $(31,32)$. However, as no clear information is available about the course of inflammation, it is not possible to foresee differences in clinical progression in advance. Therefore, it is considered that the clarification of the diagnosis of ACO is crucial for follow-up and progression.

A limitation of the present study may be that an asthma group was not included as a third group. With the inclusion of this third group, the data of the three groups (COPD-asthma-ACO) could have been evaluated for a better comparison. Nevertheless, the differential diagnosis of asthma was not included as it can be made more easily and more clearly than the other two disease groups due to its reversibility, the absence of persistent airway obstruction and a history of allergy-atopy. In studies related to ACO, its evaluation with COPD has been further discussed. In a study conducted on cytokine and interleukin (IL) levels in this regard, in which the inflammatory response was evaluated, it was reported that the inflammatory response in ACO was similar to that in COPD (17). In another study conducted on this topic, the mean NLR value was found to be significantly higher in asthmatic patients compared with the controls, and it was stated that the NLR increased as the asthma control status worsened. It was also reported that NLR, together with the asthma control test (ACT), is a guide for evaluating asthma control status (18). IL-6 cannot be easily evaluated in daily practice (as its evaluation is associated with high costs); however, it is significant for ACO distinction; the IL-6 levels have been found to be higher in ACO than in COPD and asthma (33). In the present study, the IL-6 levels could not be measured since they were not examined at the center (Ahi Evran University Research and Training Hospital, Kirsehir, Turkey). Thus, this may not be a practical application since it cannot be studied in every center.

Another limitation of the present study may be the limited number of patients used. The reason for this was that the study coincided with the COVID-19 pandemic period and patient inclusion was limited, and the inclusion criteria were multi-parameter (exclusion of patients with additional diseases and drug use, inclusion of patients with a smoking history and radiological findings) in order to exclude additional inflammatory factors. Studies with larger samples would of course yield more optimal results; however, the data of the present study were presented in the current form in order to provide insight COPD and ACO and to provide a practical approach for patient follow-up.

Consequently, the role of inflammation in ACO appears to be somewhat complex and has not been fully resolved. However, it seems that eosinophilia in ACO does not seem to be very significant in the differential diagnosis of COPD. Based on the findings of the present study, MPV does not provide additional use for the diagnosis-differential diagnosis of COPD and ACO. The fact that the mean PLT and lymphocyte counts were found to be significantly higher in the ACO group than the COPD group may also be a promising result in relation to the differential diagnosis of COPD. In general, it can be stated that the course of inflammation is more benign in ACO than COPD.

On the whole, as regards the question of whether COPD and ACO can be differentiated by blood parameters, the findings of the present study demonstrate that this is possible. However, further studies based on large populations are required for a more definitive answer.

\section{Acknowledgements}

The author would like to thank Professor H. Ahmet Bircan (Department of Pulmonary Medicine, Faculty of Medicine, Süleyman Demirel University, Isparta, Turkey) for providing academic criticism for the study, Professor Serdar Baki Albayrak (Faculty of Medicine, Istanbul Aydın University, Istanbul, Turkey) for providing academic criticism for the study and Professor Özkan Görgülü (Department of Biostatistics and Medical Information, Faculty of Medicine, Ahi Evran University, Kirsehir, Turkey) for assisting in the statistical analysis and data interpretation.

\section{Funding}

No funding was received.

\section{Availability of data and materials}

The datasets used and/or analyzed during the current study are available from the corresponding author on reasonable request.

\section{Author's contributions}

The author DZ designed the study, obtained and analyzed the data, and wrote the manuscript. DZ confirms the authenticity of all the raw data. The author has read and approved the final manuscript.

\section{Ethics approval and consent to participate}

Ethics committee approval for the study was obtained from the Ahi Evran University Faculty of Medicine Clinical Research Ethics Committee with (Approval no. 2019-04/46). Verbal and 
written consent was obtained from each patient who volunteered to participate in the study.

\section{Patient consent for publication}

Not applicable.

\section{Competing interests}

The author declares that he has no competing interests.

\section{References}

1. Buist AS, McBurnie MA, Vollmer WM, Gillespie S, Burney P, Mannino DM, Menezes AM, Sullivan SD, Lee TA, Weiss KB, et al; BOLD Collaborative Research Group: International variation in the prevalence of COPD (the BOLD Study): A population-based prevalence study. Lancet 370 : 741-750, 2007.

2. Kessler R, Partridge MR, Miravitlles M, Cazzola M, Vogelmeier C, Leynaud D and Ostinelli J: Symptom variability in patients with severe COPD: A pan-European cross-sectional study. Eur Respir J 37: 264-272, 2011

3. Montes de Oca M, Perez-Padilla R, Tálamo C, Halbert RJ, Moreno D, Lopez MV, Muiño A, José Roberto BJ, Valdivia G, Pertuzé J, et al; PLATINO Team: Acute bronchodilator responsiveness in subjects with and without airflow obstruction in five Latin American cities: The PLATINO study. Pulm Pharmacol Ther 23: 29-35, 2010.

4. Global Initiative for Asthma (GINA): Diagnosis of Diseases of Chronic Airflow Limitation: Asthma, COPD and Asthma-COPD Overlap Syndrome (ACOS). GINA, Fontana, WI, 2014. http://www.goldcopd.org.

5. Şen E, Oğuzülgen K, Bavbek S, Günen H, Kıyan E, Türktaş H, Yorgancioğlu A, Polatlı M, Y1ldız F, Celik G, et al: Asthma-COPD overlap syndrome. Tuberk Toraks 63: 265-277, 2015 (In Turkish).

6. Mauad T and Dolhnikoff M: Pathologic similarities and differences between asthma and chronic obstructive pulmonary disease. Curr Opin Pulm Med 14: 31-38, 2008.

7. Wurst KE, Kelly-Reif K, Bushnell GA, Pascoe S and Barnes N: Understanding asthma-chronic obstructive pulmonary disease overlap syndrome. Respir Med 110: 1-11, 2016.

8. Inoue $\mathrm{H}$, Nagase $\mathrm{T}$, Morita $\mathrm{S}$, Yoshida $\mathrm{A}$, Jinnai $\mathrm{T}$ and Ichinose M: Prevalence and characteristics of asthma-COPD overlap syndrome identified by a stepwise approach. Int J Chron Obstruct Pulmon Dis 12: 1803-1810, 2017.

9. Bateman ED, Reddel HK, van Zyl-Smit RN and Agusti A: The asthma-COPD overlap syndrome: Towards a revised taxonomy of chronic airways diseases? Lancet Respir Med 3: 719-728, 2015.

10. Global Initiative for Asthma (GINA): Global Strategy for Asthma Management and Prevention. GINA, Fontana, WI, 2014. https:// ginasthma.org/wp-content/uploads/2019/01/2014-GINA.pdf.

11. Zeki AA, Schivo M, Chan A, Albertson TE and Louie S: The asthma-COPD overlap syndrome: A common clinical problem in the elderly. J Allergy (Cairo) 2011: 861926, 2011.

12. Gibson PG and Simpson JL: The overlap syndrome of asthma and COPD: What are its features and how important is it? Thorax 64: 728-735, 2009.

13. Terradas R, Grau S, Blanch J, Riu M, Saballs P, Castells X, Horcajada JP and Knobel H: Eosinophil count and neutrophil-lymphocyte count ratio as prognostic markers in patients with bacteremia: a retrospective cohort study. PLoS One 7: e42860, 2012.

14. Dirican N, Anar C, Kaya S, Bircan HA, Colar HH and Cakir M: The clinical significance of hematologic parameters in patients with sarcoidosis. Clin Respir J 10: 32-39, 2016.

15. Akyüz A, Akkoyun DÇ, Oran M, Değirmenci H and Alp R: Mean platelet volume in patients with obstructive sleep apnea and its relationship with simpler heart rate derivatives. Cardiol Res Pract 2014: 454701, 2014.

16. Bülbül Y, Aydın Özgür E and Örem A: Platelet indices in obstructive sleep apnea: The role of mean platelet volume, platelet distribution widht and plateletcrit. Tuberk Toraks 64: 206-210, 2016.
17. Karadeniz G, Aktoğu S, Erer OF, Kır SB, Doruk S, Demir M and Sonat K: Predictive value of platelet-to-lymphocyte ratio in exacerbation of chronic obstructive pulmonary disease. Biomark Med 10: 701-710, 2016.

18. Çoban A ğca M, Aksoy E, Duman D, Özmen İ, Yıldırım E, Güngör S, Durmus N, Aka Aktürk Ü,Saltürk C, Tepetam FM, et al: Does eosinophilia and neutrophil to lymphocyte ratio affect hospital re-admission in cases of COPD exacerbation? Tuberk Toraks 65: 282-290, 2017

19. de Marco R, Pesce G, Marcon A, Accordini S, Antonicelli L, Bugiani M, Casali L, Ferrari M, Nicolini G, Panico MG, et al: The coexistence of asthma and Chronic Obstructive Pulmonary Disease (COPD): prevalence and risk factors in young, middle-aged and elderly people from the general population. PLoS One 8: e62985, 2013.

20. Hardin M, Silverman EK, Barr RG, Hansel NN, Schroeder JD, Make BJ, Crapo JD and Hersh CP; COPDGene Investigators: The clinical features of the overlap between COPD and asthma. Respir Res 12: 127, 2011.

21. Zorlu D, Dirican A, Bayiz H, Uzar T, Topbasi N and Ozkaya S: Asthma, asthma-COPD overlap syndrome and chronic obstructive pulmonary disease in Turkey. Minerva Pneumol 57: 68-72, 2018.

22. Uchida A, Sakaue K and Inoue H: Epidemiology of asthma-chronic obstructive pulmonary disease overlap (ACO). Allergol Int 67: 165-171, 2018.

23. Soler-Cataluña JJ, Cosío B, Izquierdo JL, López-Campos JL, Marín JM, Agüero R, Baloira A, Carrizo S, Esteban C, Galdiz JB, et al: Consensus document on the overlap phenotype COPD-asthma in COPD. Arch Bronconeumol 48: 331-337, 2012.

24. Louie S, Zeki AA, Schivo M, Chan AL, Yoneda KY, Avdalovic M, Morrissey BM and Albertson TE: The asthma-chronic obstructive pulmonary disease overlap syndrome: pharmacotherapeutic considerations. Expert Rev Clin Pharmacol 6: 197-219, 2013.

25. Bujarski S, Parulekar AD, Sharafkhaneh A and Hanania NA: The asthma COPD overlap syndrome (ACOS). Curr Allergy Asthma Rep 15: 509, 2015.

26. Soriano JB, Davis KJ, Coleman B, Visick G, Mannino D and Pride NB: The proportional Venn diagram of obstructive lung disease: Two approximations from the United States and the United Kingdom. Chest 124: 474-481, 2003.

27. Papaiwannou A, Zarogoulidis P, Porpodis K, Spyratos D, Kioumis I, Pitsiou G, Pataka A, Tsakiridis K, Arikas S, Mpakas A, et al: Asthma-chronic obstructive pulmonary disease overlap syndrome (ACOS): Current literature review. J Thorac Dis 6 (Suppl 1): S146-S151, 2014.

28. Yoshida M, Nakano T, Fukuyama S, Matsumoto T, EguchiM, Moriwaki A, Takata S, Machida K, Kanaya A,Matsumoto K, et al: Effects of tiotropium on lung function in severe asthmatics with or without emphysematous changes. Pulm Pharmacol Ther 26: 159-166, 2013.

29. Song JH, Lee CH, Kim JW, Lee WY, Jung JY, Park JH, Jung KS, Yoo KH, Park YB and Kim DK: Clinical implications of blood eosinophil count in patients with non-asthma-COPD overlap syndrome COPD. Int J Chron Obstruct Pulmon Dis 12: 2455-2464, 2017.

30. Hikichi M, Hashimoto S and Gon Y: Asthma and COPD overlap pathophysiology of ACO. Allergol Int 67: 179-186, 2018.

31. Izbicki G, Teo V, Liang J, Russell GM, Holland AE, Zwar NA, Bonevski B, Mahal A, Eustace P, Paul E, et al: Clinical Characteristics Of Patients With Asthma COPD Overlap (ACO) In Australian Primary Care. Int J Chron Obstruct Pulmon Dis 14: 2745-2752, 2019.

32. Yanagisawa S and Ichinose M: Definition and diagnosis of asthma-COPD overlap (ACO). Allergol Int 67: 172-178, 2018

33. Fu JJ, McDonald VM, Gibson PG and Simpson JL: Systemicinflam-mation in older adults with asthma-COPD overlap syndrome. Allergy Asthma Immunol Res 6: 316-324, 2014.

This work is licensed under a Creative Commons Attribution-NonCommercial-NoDerivatives 4.0 International (CC BY-NC-ND 4.0) License. 